\title{
Transplantation of mesenchymal stem cells induced into neuronlike cells by sodium ferulate
}

\author{
Zhifeng Deng ${ }^{1}$, Wei Tu ${ }^{1}$, Yang Wang ${ }^{2}$, Yuanlei Lou ${ }^{2}$ \\ ${ }^{I}$ Department of Neurosurgery, the Second Affiliated Hospital of Nanchang University; ${ }^{2}$ Institute of Urology,Nanchang University, \\ Nanchang 330006, China
}

Mesenchymal stem cells (MSCs) are multipotent cells which can be differentiated into neural cells and provide an excellent cell source for neural regeneration and repair. However, recent reports showed that few transplanted MSCs adopted neural cell fate in rat brain after stroke. Ex vivo neural induction of MSCs before transplantation may be an alternative therapy for stroke. Our previous studies demonstrated that MSCs can be differentiated into neuron-like cells induced by sodium ferulate in vitro. In this study, we investigated the survival and distribution of MSCs which induced into neuron-like cells by sodium ferulate in the brain of MCAO rats after transplantation. Human MSCs were isolated from bone marrow by gradient centrifugation, and purified by adherent culture. The third passage of MSCs were induced with sodium ferulate for $24 \mathrm{~h}$ after pre-induced by bFGF for $24 \mathrm{~h}$. For cellular identification, MSCs were prelabeled with fluorescent dye CM-Di1. Rats were subjected to $2 \mathrm{~h}$ of middle cerebral artery occlusion (MCAO), received grafts at $24 \mathrm{~h}$ and were euthanized at $14 \mathrm{~d}$ after MCAo. The frozen sections of rat brain were examined by fluorescence microscopy and immunofluorescence with an antibody specific for neuron. The results showed that differentiated cells induced by sodium ferulate for $24 \mathrm{~h}$ showed typical neural morphology with contracted cell body and extended long processes. After intravenous MSCs administration, MSCs were found to be survived in damaged brain tissue, and more survived cells were found in the ischemic side of brain than in the contralateral non-ischemic side. Immunofluorescent staining showed some MSCs expressed the neuron special enolase(NSE). The data indicate that MSCs induced by sodium ferulate can survive in the brain of MCAO rats and remained the differentiation properts to neuron cells.

Keywords: mesenchymal stem cells, sodium ferulate, induction, transplantation

Cell Research (2008) 18:s136. doi: 10.1038/cr.2008.226; published online 4 August 2008

Correspondence: Zhifeng Deng

E-mail: dengzf63@126.com

National Natural Science Foundation of China (No. 30560156); Natural

Science Foundation of Jiangxi province (No. 0540087) 